\title{
An acoustic waveguide using doubly-bonded silicon/thinned PPT/silicon structures for RF applications
}

\author{
F. Bassignot, E. Courjon, G. Ulliac, T. Laroche, J. \\ Garcia, S. Queste, J-P. Romand, S. Ballandras \\ Institut FEMTO-ST \\ UMR 6174 CNRS-UFC-ENSMM-UTBM \\ 26 che. de l'épitaphe, 25000 Besançon, France \\ sylvain.ballandras@,femto-st.fr
}

\author{
R. Petit \\ CELAR \\ DGA \\ Rennes, France
}

\begin{abstract}
In this paper, we present new results on the development of piezoelectric transducers based on periodically poled ferroelectric domains in a lithium niobate plate bonded between two silicon wafers. The fabrication of the periodically poled transducers operating in the range $50-500 \mathrm{MHz}$ has been achieved on a 3 inches 500 mm thick wafer. These devices then have been bonded on silicon wafers to fabricate a waveguide. Guided elliptic as well as partially guided longitudinal modes are excited. The experimental responses of the tested devices are compared to predicted harmonic admittances, showing a good agreement between both results and allowing for a reliable analysis of the nature of the excited modes. We also show interesting studies of material combinations used to guide ultrasonic waves. Dispersion properties have also been studied for a structure Si/PPT/Si. Operating points corresponding to a specific thickness/period ratio are found. Therefore a new conception with a Si/thinned PPT/Si structure is fabricated.
\end{abstract}

Keywords-component: Periodically poled Lithium Niobate; FEA-BEAM; waveguide; dispersion behaviour; isolated wave

\section{INTRODUCTION}

Surface Acoustic Waves resonators may be used for the frequency stabilization of electrical oscillators until a few Gigahertzes $(\mathrm{GHz})$. In some cases, these resonators are limited by technical and compactness specifications imposed by radar applications. Consequently, passive components using guided acoustic waves are well-suited for such an application. Periodically poled transducers (PPTs) have been investigated recently as an alternative to classical inter-digital transducers for the excitation and detection of guided acoustic waves. We propose here a new compact structure allowing high frequency operation with a simplified package as the wave is trapped in a sandwich structure based on $\mathrm{Si} / \mathrm{LiNbO} / \mathrm{Si}$ material combination. We investigate the capability of such devices to answer the above specifications.

We explain the PPTs fabrication principle and present experimental results on 3" and 4" $\mathrm{LiNbO} 3$ and $\mathrm{LiTaO} 3$ wafers. We then report theoretical analysis results of the PPT operation obtained, using our finite and boundary element simulation tool. Thanks to the analysis of the transducer dispersion properties providing the optimum operating points and the temperature coefficient of frequency (TCF) analysis, we have chosen the best combination $\mathrm{Si} / \mathrm{PPT} / \mathrm{Si}$ to fabricate test vehicles. Poled $\mathrm{LiNbO} 3$ wafers are first bonded on silicon (Si) using $\mathrm{Au} / \mathrm{Au}$ compression, then thinned down to a few dozen microns. The resulting stack is metalized to process a second wafer bonding on Si. These devices exhibit a $50 \mu \mathrm{m}$ period, yielding operating frequencies near $80 \mathrm{MHz}$. We have pointed out the existence of elliptically polarized guided modes for various material combinations and technologicalparameters. We also have identified the possibility to excite partially guided longitudinally polarized waves. The control of the ferroelectric material thickness has been achieved (lapping and polishing). The comparison between theoretical and experimental TCF shows that we are able to accurately predict the thermo-elastic behavior of the material stack.

\section{A NEW WAVEGUIDE CONCEPT: SUBSTRATE/PPT/SUBSTRATE :}

In this part, we will first present a new waveguide concept. We present then the theoretical results achieved using our finite element/boundary element simulation tool [1], allowing the simulation of such material combinations. After that, the combination of material $\mathrm{Si} / \mathrm{PPT} / \mathrm{Si}$ is chosen (the easiest and the cheapest structure to fabricate) and the dispersion properties of this transducer are studied providing the best operating points.

\section{A. New waveguide concept}

An innovative solution has been retained in order to answer to the need of high frequencies sources (RF until $\mathrm{X}$ band domain). These sources indeed need spectral purity, short-term stability and phase noise around the carrier wave. The purpose is to fabricate resonators based on the ultrasonic waves propagation through a waveguide. This resonator operates with a PPT structure inserted between two guiding substrates. A wave propagates without any acoustic losses and decreases exponentially in the structure (fig. 1). This definition is similar in these interface waves [2]. Nevertheless, the principle is substantially different for various reasons. First, the transduction structure corresponds to a layer in which ferroelectric domains have been artificially structured as 
developed in [3-4-5]. Classical interdigital transducers have been given up in this kind of structure because the bonding is difficult due to the electrode pattern. In this case, a polymer film is deposited between both substrates to be bonded in order to create an elastic link introducing important acoustic losses.

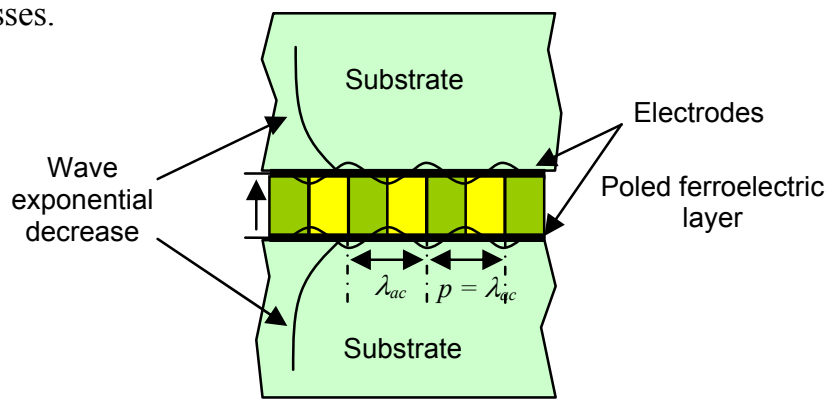

Figure 1. Scheme of the new concept waveguide based on PPT

\section{B. Analysis of several material combinations}

The purpose of this analysis is to study influences on wave guidance and velocity. For that, we study two principal cases using $\mathrm{LiNbO}_{3}$ and $\mathrm{LiTaO}_{3}$ as ferroelectric material and we modify the guidance material (silicon, sapphire or diamond). Theoretical analysis results of these structures are obtained by means of our finite and boundary element simulation tool. Figures 2 and 3 show the theoretical admittance of $\mathrm{LiNbO}_{3}$ and $\mathrm{LiTaO}_{3}$ PPT respectively $(\mathrm{p}=50 \mu \mathrm{m})$.

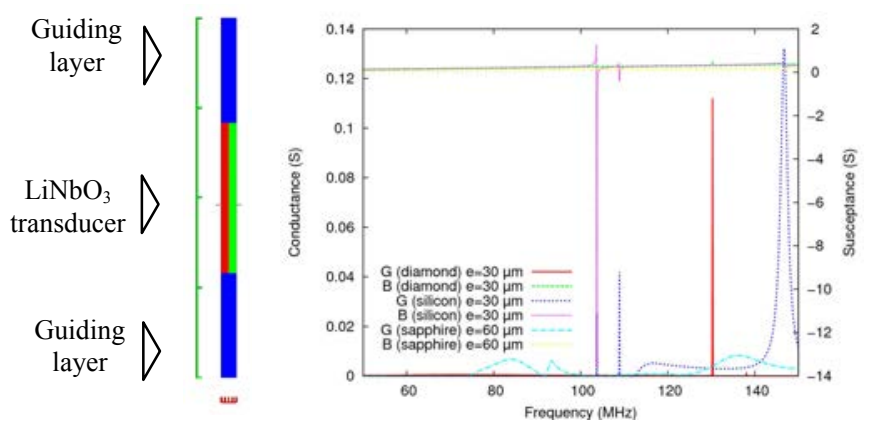

(a)

(b)

Figure 2.

(a) Scheme of the PPT structure (b) Theoretical admittance of a $50 \mu \mathrm{m}$ period $\mathrm{LiNbO}_{3}$ PPT for 30 or $60 \mu \mathrm{m}$ thick for different guiding substrates

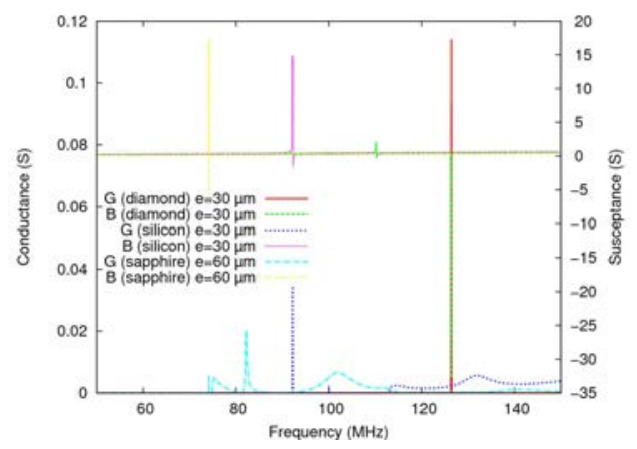

Figure 3. Theoretical admittance of a $50 \mu \mathrm{m}$ period $\mathrm{LiTaO}_{3} \mathrm{PPT}$ for 30 or $60 \mu \mathrm{m}$ thick for different guiding substrates
There are two main differences between $\mathrm{LiNbO}_{3}$ and $\mathrm{LiTaO}_{3}$ transducers. The first one is a better spectrum purity for the $\mathrm{LiTaO}_{3}$ configuration in comparison with the $\mathrm{LiNbO}_{3}$ configuration. The second one concerns the Rayleigh wave velocity of $\mathrm{LiTaO}_{3}\left(3500 \mathrm{~m} . \mathrm{s}^{-1}\right)$ which is lower than the one of $\mathrm{LiNbO}_{3}\left(3800 \mathrm{~m} . \mathrm{s}^{-1}\right)$, that is why we observe the same acoustic waves for both materials and for each guiding substrates but at a lower frequency for the $\mathrm{LiTaO}_{3}$. The guidance conditions also depend on the ratio between the ferroelectric material thickness and the transducer wavelength $(\mathrm{h} / \mathrm{p})$. The elliptic mode actually is guided from a ratio $\mathrm{h} / \mathrm{p}$ equal to 1 . The structure diamond/PPT/diamond is the most guiding configuration thanks to the high propagation velocities in the diamond (12500 $\mathrm{m} \cdot \mathrm{s}^{-1}$ for the Surface Skimming Bulk Wave (SSBW)). In fact, the quasi-longitudinal mode is excited and guided at a velocity around $6500 \mathrm{~m} \cdot \mathrm{s}^{-1}$. The last structure consisting of a silicon/PPT/silicon stuck presents interesting guidance properties for a ratio $(\mathrm{h} / \mathrm{p})$ smaller than 1 , this parameter is treated in the next part. In this structure, the elliptic mode is mainly excited at about $5100 \mathrm{~m} \cdot \mathrm{s}^{-1}$ whereas the longitudinal mode at about $7300 \mathrm{~m} . \mathrm{s}^{-1}$ is not guided, as the SSBW velocity in the silicon is near $5800 \mathrm{~m} \cdot \mathrm{s}^{-1}$. This configuration is retained for the rest of the study because the elliptic mode is well guided, the fabrication process is quite simple and cheaper than structures based on other guiding materials.

\section{Analysis of dispersion properties}

We propose to closely investigate how to efficiently derive the dispersion curves and then to show at least for the elliptic mode of the waveguide the influence of the PPT geometry and the operating frequency on the mode properties. In that matter, we have considered the general shape of the transducer symbolized in fig.4. We assume here a propagation between two silicon layers but the approach can be generalized to any guiding substrate. The period is arbitrarily fixed to $50 \mu \mathrm{m}$ (i.e. width of each $\mathrm{LiNbO}_{3}$ domain set to $25 \mu \mathrm{m}$, poling ratio $=0.5$ ).

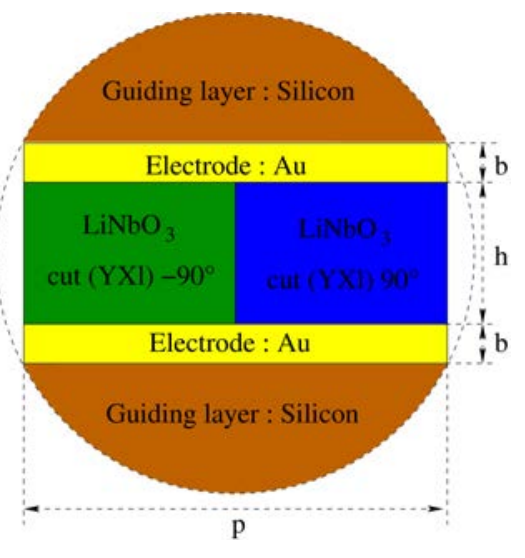

Figure 4. Scheme of PPT principle and generic shape for dispersion curve derivation

In Figure 5, we have plotted the theoretical admittance (conductance and susceptance) versus frequency for a thickness $/$ period $(\mathrm{h} / \mathrm{p})$ ratio equal to 0.1 , in order to identify the main contribution corresponding to an elliptically shaped mode. 


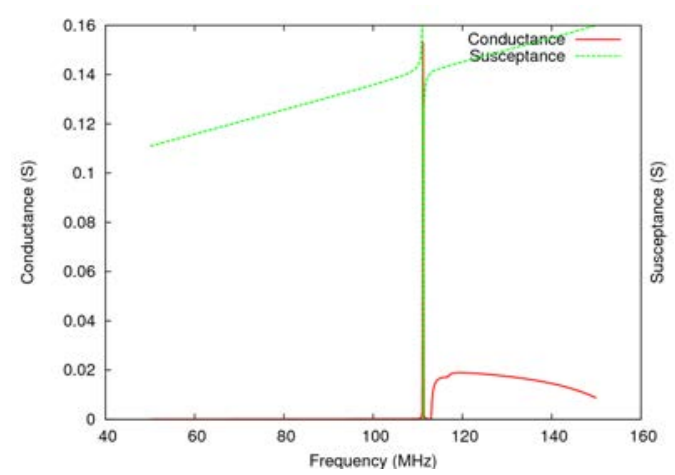

Figure 5. Harmonic admittance of the structure plotted in fig.4 for a thickness/period ratio equal to 0.1

Once the mode identified, we follow the evolution of its resonance frequency and electromagnetic coupling coefficient for $\mathrm{h} / \mathrm{p}$ ratios ranging from 0.1 to 10 , is plotted on fig. 6 .

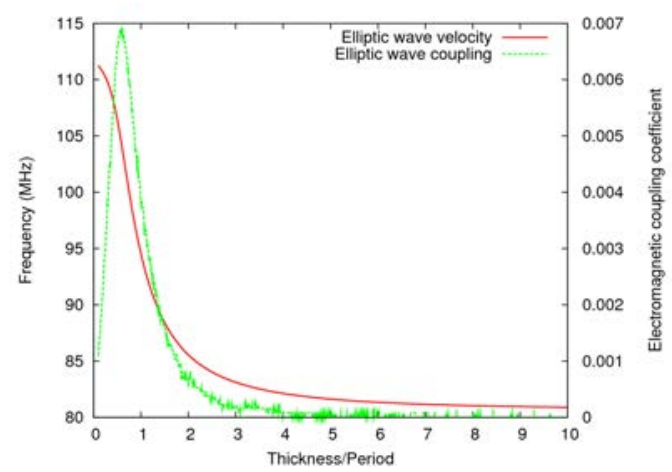

Figure 6. Evolution of the elliptic mode synchronism frequency (red curve) and electromagnetic coupling (green curve) for small values of $\mathrm{h} / \mathrm{p}$ ratio (ranging from 0.1 to 10 in our calculation)

There is no ambiguity concerning the elliptic polarization of the studied mode because there is no possible interaction with other modes (as often observed for Lamb waves for instance). This operating point is of very particular importance as it conditions the possibility to improve the design reliability by choosing operation condition poorly affected by technology, which is a key point for any practical exploitation of the device. The next section shows the different technological steps of a device $\mathrm{Si} / \mathrm{PPT} / \mathrm{Si}$ fabrication.

\section{EXPERIMENTAL VALIDATION OF THE CONCEPT SI/PPT/SI AND CHARACTERIZATION}

The concept has been experimentally checked first using a thick structure consisting of a $\operatorname{Silicon}(380 \mu \mathrm{m}$ thick $) / \mathrm{LiNbO}_{3}$ PPT $(500 \mu \mathrm{m}) /$ Silicon $(380 \mu \mathrm{m})$ material stack.

\section{A. Fabrication}

The fabrication of PPT-based waveguides consists in bonding a silicon wafer on each side of the periodically poled wafer. In that purpose, the lithium niobate wafer is bonded on a (100) 3" silicon wafer using a wafer bonding technique developed in our group based on a metal-metal adhesion at room temperature promoted by a high pressure applied to the

material stack (fig.7b). Connection via are etched in each silicon substrate by Deep Reactive Ion Etching (DRIE) in order to reach the buried metallic electrodes. After that, the structure is diced to obtain 5 separated devices. The process flowchart is reported in fig. 7 a, showing the different implemented coatings and the technological sequence.

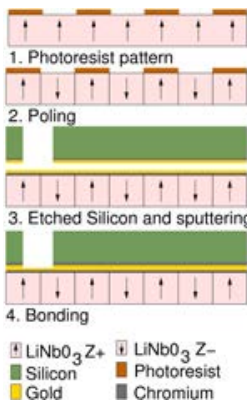

Figure 7. fabrication (b) Double bonding of a lithium niobate wafer with tw
wafers using room temperature $\mathrm{Au} / \mathrm{Au}$ bonding process

A test vehicle is mounted on a metallic surface (printed circuit) to connect electrically the structure with the support and a SMA port is added to connect the support to a RF spectrum analyzer (fig.8).

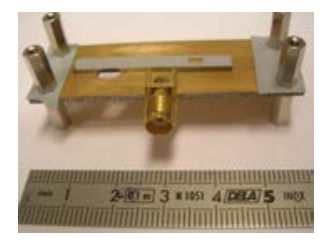

Figure 8.

Test vehicle of a $\mathrm{Si} / \mathrm{LiNbO}_{3} \mathrm{PPT} / \mathrm{Si}$ device

\section{B. Characterization and temperature sensibility}

Figure 9 presents the comparison between the experimentally measured responses of the implemented devices and the theoretical harmonic admittances obtained with our periodic finite element code. Measurements have been achieved successfully for the $50 \mu \mathrm{m}$ period devices, as explained above. Since the implemented single-port test devices are quite long and almost behave as single port resonators, the comparison between measurement and harmonic admittance results makes sense.

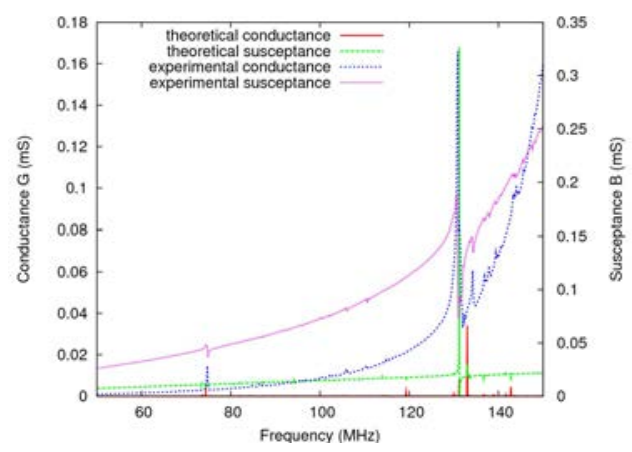

Figure 9. Comparison between the test vehicle admittance and the theoretical admittance 
This plot points out the good agreement between both results. We find two main contributions corresponding to the elliptical mode and the longitudinal mode. We can notice that the experimental admittance increases gradually. The main cause seems to be the wire length of the connection between the device and the support. This proves the feasibility of $\mathrm{LiNbO}_{3}$-based Silicon/PPT/Silicon devices.

We also have measured the experimental admittance of the longitudinally polarized wave at different temperatures (between 278 and $323 \mathrm{~K}$ ) of the test vehicle. The theoretical and the experimental Temperature Coefficient of Frequency (TCF) are in the same order of magnitude at around $80 \mathrm{ppm} / \mathrm{K}$. We can notice that the TCF is sensibly the same than the one of isolated $\mathrm{LiNbO}_{3}$ devices. So the waveguide concept based on a double silicon bonding on a thick PPT substrate (about $500 \mu \mathrm{m}$ ) has been validated. Now, the purpose is to use the ultrasonic wave excited to do a resonator for instance. Thus, following the dispersion properties modelling for a thin PPT layer, the wave is well coupled and the Q factor is high. So that, the next part presents the fabrication of a $\mathrm{Si} /$ thinned PPT/Si waveguide.

\section{DOUBLE BONDING SI/THINNED PPT/SI : FABRICATION AND CHARACTERIZATION}

The analysis of the dispersion properties of a device constituted by $\mathrm{Si} / \mathrm{PPT}(\lambda=50 \mu \mathrm{m}) / \mathrm{Si}$ has shown a specific operating point for which a single wave is excited with a maximum electromagnetic coupling.

\section{A. Fabrication}

Technological steps have already been experimentally checked using a thick structure consisting of a Silicon $(380 \mu \mathrm{m}$ thick $) / \mathrm{LiNbO}_{3}$ PPT $(500 \mu \mathrm{m}) /$ Silicon $(380 \mu \mathrm{m})$ material stack. The study of the dispersion properties enables to define a specific configuration using a thinned PPT layer of about $30 \mu \mathrm{m}$. The thinning down is achieved by home-made lapping and polishing techniques. However, the layer obtained can have surface defaults due to dust presence at the bonding interface. Consequently, the structure can only be partly bonded.

\section{B. Characterization and comparison with theory}

Test vehicles have been achieved and characterized with a network analyzer. Figure 10 presents the comparison between the measured responses of the implemented devices and the theoretical harmonic admittances obtained with our periodic finite element code. Measurements have been achieved successfully for the $50 \mu \mathrm{m}$ period devices, as explained above. Since the implemented single-port test devices are quite long and almost behave as single port resonators, the comparison between measurement and harmonic admittance results makes sense.

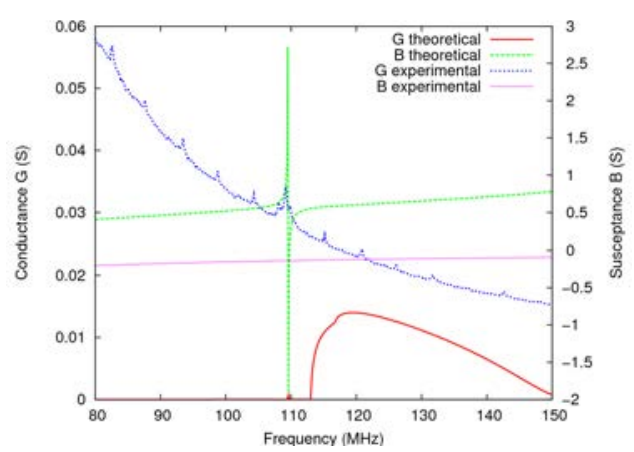

Figure 10. Comparison between the test vehicle admittance and the theoretical admittance

This plot points out the good agreement between both results. We find only one contribution (except bulk waves) corresponding to the isolated wave. We can notice that the experimental admittance decreases gradually as explained above for a thick structure. The parasitic bulk waves seem to come from the electrode shape which is bigger than the transducer.

\section{CONCLUSION}

We have pointed out the existence of elliptically polarized guided modes for various material combinations and technological parameters. We also have identified the possibility to excite partially guided longitudinally polarized waves. The control of the ferroelectric material thickness has been achieved (lapping and polishing). Several devices have been successfully fabricated with different thicknesses and tested. As predicted, we have measured both contributions when the PPT is thick and only one contribution when the PPT is thinned. Besides, we prove that the final device can be packaged easily in a low cost housing.

\section{ACKNOWLEDGMENT}

This work was supported by the french DGA (Délégation Générale pour l'Armement) under grant\#07-34-020.

\section{REFERENCES}

[1] S. Ballandras, R. Lardat, M. Wilm, Th. Pastureaud, A. Reinhardt, N. Champavert, W. Steichen, W. Daniau, V. Laude, R. Armati, G. Martin, "A mixed finite element/boundary element approach to simulate complex guided elastic wave periodic transducers", accepted for publication in Journal of Applied Physics, 2008

[2] H. Majjad, D. Gachon, V. Laude, S. Ballandras, "Interface acoustic wave devices made by indirect bonding of lithium niobate on silicon", Proc. of the IEEE US, 2006

[3] E. Courjon, J. Hauden, J. Masson, D. Gachon, L. Gauthier Manuel, W. Daniau, N. Bodin, S. Ballandras, "Pure longitudinal plate mode excited by poled domains transducers on $\mathrm{LiNbO}_{3}$ ", Proc. of the IEEE IFCS, 2007

[4] E. Courjon, J. Hauden, P. Parruch, J. Masson, D. Gachon, L. Gauthier Manuel, W. Daniau, , N. Bodin, J.M. Triscone, S. Ballandras, "Fabrication of periodically poled domains transducers on $\mathrm{LiNbO}_{3}$ ", Proc. of the IEEE IFCS, Miami, 2006

[5] S. Ballandras, W. Daniau, B. Gautier, D. Hauden, M. Wilm, V. Laude, V. Ruch, S.Flambart, "A novel surface wave transducer based on periodically poled piezoelectric domain", Proc of the IEEE EFTF-IFCS, 2003 